Virginia Commonwealth University vCU Scholars Compass

2014

Direct observation of metal nanoparticles as heterogeneous nuclei for the condensation of supersaturated organic vapors: Nucleation of sizeselected aluminum nanoparticles in acetonitrile and n-hexane vapors

Victor Abdelsayed

Virginia Commonwealth University

M. Samy El-Shall

Virginia Commonwealth University, mselshal@vcu.edu

Follow this and additional works at: http://scholarscompass.vcu.edu/chem_pubs

Part of the Chemistry Commons

Abdelsayed, V., and El-Shall, M. S. Direct observation of metal nanoparticles as heterogeneous nuclei for the condensation of supersaturated organic vapors: Nucleation of size-selected aluminum nanoparticles in acetonitrile and nhexane vapors. The Journal of Chemical Physics, 141, 054710 (2014). Copyright (C) 2014 AIP Publishing LLC.

Downloaded from

http://scholarscompass.vcu.edu/chem_pubs/67

This Article is brought to you for free and open access by the Dept. of Chemistry at VCU Scholars Compass. It has been accepted for inclusion in Chemistry Publications by an authorized administrator of VCU Scholars Compass. For more information, please contact libcompass@vcu.edu. 


\title{
Direct observation of metal nanoparticles as heterogeneous nuclei for the condensation of supersaturated organic vapors: Nucleation of size-selected aluminum nanoparticles in acetonitrile and $n$-hexane vapors
}

\author{
Victor Abdelsayed ${ }^{\mathrm{a})}$ and M. Samy El-Shall ${ }^{\mathrm{b})}$ \\ Department of Chemistry, Virginia Commonwealth University, Richmond, Virginia 23284-2006, USA
}

(Received 15 January 2014; accepted 8 July 2014; published online 5 August 2014)

\begin{abstract}
This work reports the direct observation and separation of size-selected aluminum nanoparticles acting as heterogeneous nuclei for the condensation of supersaturated vapors of both polar and nonpolar molecules. In the experiment, we study the condensation of supersaturated acetonitrile and n-hexane vapors on charged and neutral $\mathrm{Al}$ nanoparticles by activation of the metal nanoparticles to act as heterogeneous nuclei for the condensation of the organic vapor. Aluminum seed nanoparticles with diameters of 1 and $2 \mathrm{~nm}$ are capable of acting as heterogeneous nuclei for the condensation of supersaturated acetonitrile and hexane vapors. The comparison between the Kelvin and Fletcher diameters indicates that for the heterogeneous nucleation of both acetonitrile and hexane vapors, particles are activated at significantly smaller sizes than predicted by the Kelvin equation. The activation of the $\mathrm{Al}$ nanoparticles occurs at nearly $40 \%$ and $65 \%$ of the onset of homogeneous nucleation of acetonitrile and hexane supersaturated vapors, respectively. The lower activation of the charged $\mathrm{Al}$ nanoparticles in acetonitrile vapor is due to the charge-dipole interaction which results in rapid condensation of the highly polar acetonitrile molecules on the charged Al nanoparticles. The charge-dipole interaction decreases with increasing the size of the $\mathrm{Al}$ nanoparticles and therefore at low supersaturations, most of the heterogeneous nucleation events are occurring on neutral nanoparticles. No sign effect has been observed for the condensation of the organic vapors on the positively and negatively charged Al nanoparticles. The present approach of generating metal nanoparticles by pulsed laser vaporization within a supersaturated organic vapor allows for efficient separation between nucleation and growth of the metal nanoparticles and, consequently controls the average particle size, particle density, and particle size distribution within the liquid droplets of the condensing vapor. Strong correlation is found between the seed nanoparticle's size and the degree of the supersaturation of the condensing vapor. This result and the agreement among the calculated Kelvin diameters and the size of the nucleating $\mathrm{Al}$ nanoparticles determined by transmission electron microscopy provide strong proof for the development of a new approach for the separation and characterization of heterogeneous nuclei formed in organic vapors. These processes can take place in the atmosphere by a combination of several organic species including polar compounds which could be very efficient in activating charged nanoparticles and cluster ions of atmospheric relevance. () 2014 AIP Publishing LLC. [http://dx.doi.org/10.1063/1.4890726]
\end{abstract}

\section{INTRODUCTION}

Nucleation is the formation of a new phase from a continuous phase in which small clusters of atoms or molecules organize into nuclei, which can occur homogeneously and/or heterogeneously. ${ }^{1-3}$ It is one of the most ubiquitous and important phenomena in science and technology. It plays a central role not only in materials science, metallurgy, crystal growth, and chemical processing, but also in aerosol formation, wind tunnels, atmospheric science, cosmochemistry, bone formation, protein crystallization, and understanding of blood disorders such as sickle cell anemia. ${ }^{1-7}$

\footnotetext{
a) Present address: URS Corporation, National Energy Technology Laboratory (NETL), U.S. Department of Energy, 3610 Collins Ferry Road, Morgantown, West Virginia 26507-0880, USA.

b) Author to whom correspondence should be addressed. Electronic mail: mselshal@vcu.edu. Also affiliated with the Department of Chemistry, King Abdulaziz University, Jeddah 21589, Saudi Arabia.
}

Vapor phase homogeneous nucleation is the simplest form of nucleation. It involves the decay of a metastable state, i.e., a supersaturated vapor, by the spontaneous occurrence of large thermal fluctuations through the formation of nuclei or embryonic droplets of the liquid phase., ${ }^{2,3}$ Droplets that are larger than a critical size $\left(\mathrm{n}^{*}\right)$ grow, and thus the stable phase results. The critical cluster size $\left(n^{*}\right)$ or the nucleus for condensation is reached when the evaporation rate is equal to the attachment rate of the cluster. In nucleation experiments, the consequences of the presence of the nucleus, e.g., droplet formation, precipitation, etc., are typically observed.

Although the effects of impurities or additives on nucleation rates were identified decades ago, only a few experimental studies have provided quantitative information on the heterogeneous nucleation of supersaturated vapors on size-selected nanoparticles. ${ }^{5,8-15}$ Heterogeneous nucleation is typically much faster than homogeneous nucleation because 
the nucleation barrier is much lower at a surface. ${ }^{8-10}$ This is because the nucleation barrier comes from the positive surface term in the free energy equation. ${ }^{8-10}$ The heterogeneous nucleation probability depends on several parameters such as the size of the seed particles, the contact angle between the tangent of the surface of the nucleating droplet and the seed particle, and the degree of saturation of the condensing vapor. ${ }^{8-15}$ For charged seed particles, because of the strong ion-dipole and ion-induced dipole forces relative to van der Waals dispersion forces, nucleation occurs preferentially with respect to nucleation on neutral particles. ${ }^{5,12,16-18}$ In this case, the size of the particles, the contact angle between the condensing molecules and the particle, as well as the sign and the magnitude of the charge on the particle play the most important roles in determining the thermodynamics and kinetics of the process. ${ }^{5,12,16-20}$ The molecular properties of the condensing vapor molecules such as dipole moment and polarizability can also affect the nucleation process. Few studies have investigated the activation of molecular ions and charged nanoparticles for the condensation of supersaturated vapors, $5,11,12,14,15,17-20$ but direct observation of metal nanoparticles acting as heterogeneous nuclei for the condensation of organic vapors along with the separation and identification of the nucleating nanoparticles have not been reported. Herein, we report, for the first time, direct observation and separation of size-selected metal nanoparticles acting as heterogeneous nuclei for the condensation of supersaturated vapors of both polar and nonpolar molecules. Specifically, we investigate the condensation of supersaturated vapors of acetonitrile and n-hexane on size-selected Al nanoparticles formed by laser vaporization of an aluminum target within the supersaturated host vapor. The nanoparticles of selected sizes act as heterogeneous nuclei for the condensation of the host vapor thus allowing the separation and detection of nearly monodisperse $\mathrm{Al}$ nanoparticles. This also allows the simultaneous measurement of heterogeneous nucleation rates under well-defined conditions of vapor supersaturation, temperature, and carrier gas pressure. The results provide new insights on the condensation of supersaturated vapors on nanoparticles including the effects of charged particles, charge sign, and polarity of the condensing molecules on the thermodynamics and kinetics of the heterogeneous nucleation process.

\section{EXPERIMENTAL}

The current experiments are based on coupling of the LVCC (Laser Vaporization-Controlled Condensation) ${ }^{21,22}$ technique to induce nucleation of the metal nanoparticles with a thermal diffusion cloud chamber (DCC) ${ }^{12,18,19}$ to monitor the condensation of the supersaturated organic vapors. Detailed descriptions of the DCC and the principles of its operation are available in several publications, ${ }^{23,24}$ and only a brief description is given here and in Figure S1 of the supplementary material. ${ }^{25}$ The DCC is used to produce a supersaturated host vapor by diffusion through a carrier gas from a lower heated liquid pool to an upper cold plate at which the vapor molecules are condensed to form a thin liquid film. As a result, the vapor is saturated at both the bottom and top plates, and supersaturated anywhere in-between the plates along the height of the chamber (temperature, pressure, and supersaturation profiles are given in Figure S1 of the supplementary material). The supersaturation can be made as large as desired by increasing the temperature gradient between the bottom and top plates. The maximum (peak) supersaturation occurs at an elevation corresponding to $\sim 0.70$ reduced height of the DCC (known as the nucleation zone where the onset of nucleation occurs).

In the experiment, the maximum supersaturation of acetonitrile or hexane vapor in the DCC is adjusted to a value below that required for homogeneous nucleation, ${ }^{23,26}$ therefore no nucleation is observed in the chamber. For acetonitrile and n-hexane vapors at $266 \mathrm{~K}$, the critical supersaturations $\left(\mathrm{S}_{\mathrm{c}}\right.$, supersaturation required for an average nucleation rate of $1 \mathrm{drop} / \mathrm{cm}^{3} / \mathrm{s}$ ) are 4.01 and 4.20 , respectively. ${ }^{23,26} \mathrm{~A}$ single laser pulse is fired from an ArF laser (193 nm, $5 \mathrm{~ns}$ pulse width) focused on an aluminium target placed on the bottom plate of the DCC. The laser power used ranges from 25 to $185 \mathrm{~mJ} /$ pulse corresponding to power density on the Al target of $0.20-1.5 \mathrm{~W} / \mathrm{cm}^{2}$, respectively. The heights of the Al target, the ArF laser beam, the He-Ne laser beam, and the nucleation zone are $2.5,2.3,3.0$, and $3.6-3.8 \mathrm{~cm}$, respectively from the bottom plate of the DCC (see Figure S1 of the supplementary material). ${ }^{25}$

Under the laser power used in the current experiments, a single laser pulse generates about $10^{13}-10^{14}$ metal atoms (including about $10^{5}-10^{6}$ ions) which immediately condense to form neutral and charged Al nanoparticles. ${ }^{21}$ Because of the low ionization energy of the $\mathrm{Al}$ atom $(5.99 \mathrm{eV})$, the $193 \mathrm{~nm}$ $(6.42 \mathrm{eV})$ photons are efficient in generating a significant number of charged Al nanoparticles. The nanoparticles diffuse through the vapor where they may become activated in the nucleation zone (about 3.6-3.8 cm height from the bottom plate) thus resulting in the condensation of the host vapor. The rate of heterogeneous nucleation is determined by observing the forward scattering of light from liquid droplets (containing the Al nanoparticles) falling within a well-defined volume through a horizontal He-Ne laser beam traversing the chamber using a photomultiplier (PMT) and counting electronics. Figures 1(a) and 1(b) illustrate the concept of the experiment and the experimental set-up, respectively.

\section{THEORY}

Homogeneous nucleation in supersaturated vapors has been described by the Classical Nucleation Theory (CNT), ${ }^{1,3}$ where the formation free energy of a cluster is given by

$$
\Delta G_{\mathrm{hom}}(n)=-n k_{B} T \ln S+\sigma_{v, l} A_{v, l}(\mathrm{n}) .
$$

Here, $\mathrm{n}$ is the number of molecules in the cluster, $k_{B}$ is Boltzmann's constant, $T$ is the temperature, and $S$ is the supersaturation ratio $\left(S=P / P_{e}\right.$ where $P$ is the actual pressure of the vapor, and $P_{e}$ is the equilibrium or "saturation" vapor pressure at the temperature of the vapor $T$ ). The second term in Eq. (1) represents the surface free energy of forming the interface between the cluster, described as a spherical liquid droplet with a surface area $\mathrm{A}_{v, l}(\mathrm{n})$, and the vapor. This term is given by $\left[\sigma_{v, l} \mathrm{~A}_{v, l}(\mathrm{n})=4 \pi \sigma_{v, l}(3 v / 4 \pi)^{2 / 3} n^{2 / 3}\right]$ where $\sigma_{v, l}$ 


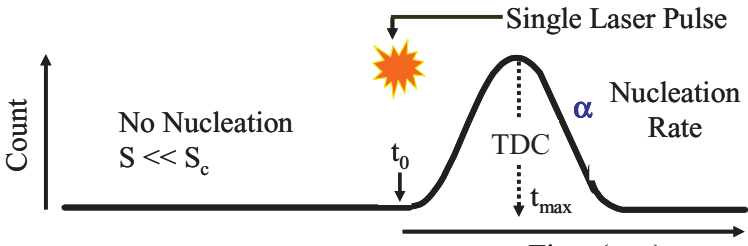

(a)

Time (sec.)

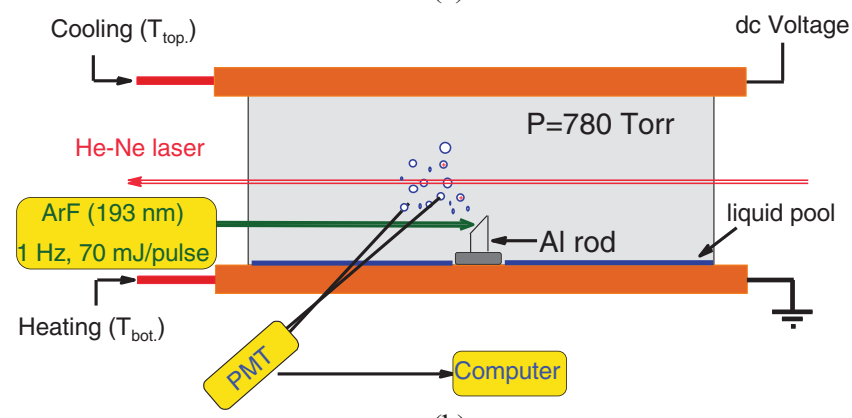

(b)

FIG. 1. (a). Schematic of the heterogeneous nucleation experiment on laser generated metal nanoparticles within a supersaturated organic vapor, (b). Experimental set-up showing the DCC with the heated and cooled bottom and top plates, respectively; the ArF laser for the generation of the Al atoms and ions; and the He-Ne laser for the detection of nucleated liquid droplets by light scattering using a photomultiplier (PMT).

is the vapor-liquid surface tension and $v$ is the volume per molecule in the bulk liquid.

The radius of the critical cluster $\left(r^{*}\right)$ is given by the Kelvin Eq. (2) and its formation free energy $\Delta \mathrm{G}^{*}{ }_{\text {hom }}$ (the barrier height for homogeneous nucleation) is given by Eq. (3) according to

$$
\begin{gathered}
r^{*}=2 v \sigma_{v, l} / k_{B} T \ln S, \\
\Delta \mathrm{G}_{\mathrm{hom}}^{*}=16 \pi\left(\sigma_{v, l}\right)^{3} v^{2} / 3\left(k_{B} T \ln S\right)^{2} .
\end{gathered}
$$

For heterogeneous nucleation, the formation free energy of a heterogeneous cluster is given by ${ }^{5,8}$

$$
\Delta G_{h e t}(n)=-n k_{B} T \ln S+\sigma_{v, l} \mathrm{~A}_{v, l}+\left(\sigma_{l, s}-\sigma_{v, s}\right) \mathrm{A}_{l, s},
$$

where $\sigma_{l, s}$ and $\sigma_{v, s}$ are the surface tensions between the liquid-seed particle $\left(\sigma_{l, s}\right)$, and the vapor-seed particle $\left(\sigma_{v, s}\right)$, respectively. $\mathrm{A}_{l, s}$ is the area of the interface between the cluster and the seed particle. According to the Fletcher theory, the critical size clusters are formed by condensation of the molecules on the seed particles which become stable for growth when their radii become similar to the Kelvin radius. $5,8,11$ The ratio $(X)$ of the radius of the spherical seed particle $\left(R_{s}\right)$ to the radius of the critical cluster $\left(r^{*}\right)$ is given by

$$
X=R_{s} / r^{*}=R_{s} k_{B} T \ln S / 2 v \sigma_{v, l} .
$$

The radius of the critical heterogeneous cluster $\left(r^{*}{ }_{h e t}\right)$ and its formation free energy $\left(\Delta \mathrm{G}^{*}{ }_{h e t}\right)$ are proportional to the radius $\left(r^{*}\right)$ and formation free energy of the homogeneous cluster $\left(\Delta \mathrm{G}^{*}{ }_{\text {hom }}\right)$, respectively, shown through Eqs. (6) and (7),

$$
\begin{gathered}
r_{\text {het }}^{*}=f_{1}(m, X) r^{*}, \\
\Delta \mathrm{G}^{*}{ }_{\text {het }}=f_{2}(m, X) \Delta \mathrm{G}_{\mathrm{hom}}^{*},
\end{gathered}
$$

where $f_{1}$ and $f_{2}$ are geometric factors that depend on $X$ and the contact angle $\theta$ between the nucleating droplet and the seed particle (through the parameter $m=\cos \theta) .^{5,8,11}$ If there is no condensation seed particle $\left(\mathrm{R}_{\mathrm{s}}=0\right)$ or if the contact between the seed and the nucleating droplet vanishes $\left(\theta \rightarrow 180^{\circ}\right), f_{1}$ and $f_{2}$ in Eqs. (6) and (7) reduce to 1, thus resulting in the homogeneous nucleation expressions (2) and (3), respectively.

In homogeneous nucleation, the critical supersaturation $\left(\mathrm{S}_{\mathrm{c}}\right)$ corresponding to a nucleation rate of $1 \mathrm{drop} / \mathrm{cm}^{3} / \mathrm{s}$ is usually taken as the onset of nucleation. ${ }^{1-3}$ In the heterogeneous case both the concentration and the size distribution of the seed particles will affect the nucleation rate. The probability $(P)$ for heterogeneous nucleation on a seed particle with radius $R_{s}$ within a time period $t$ can be defined as ${ }^{5,11}$

$$
P=1-\exp \left(-J_{h e t} 4 \pi R_{s}^{2} t\right),
$$

where $J_{h e t}$ is the heterogeneous nucleation rate. The onset of heterogeneous nucleation is then taken as the supersaturation corresponding to $P=0.5$ and the corresponding $R_{s}$ value is defined in Eq. (9) as the Fletcher radius ${ }^{5,11}$

$$
R_{F}=R_{s}(\text { for } P=0.5) \text {. }
$$

It is clear from Eq. (2) that as the supersaturation ratio increases, the critical size required for condensation decreases and, therefore, nucleation can be induced by the presence of small clusters within the supersaturated vapor. It is also clear from Eq. (7) that for all the allowed values of the condensation seed particle radius $\left(\mathrm{R}_{\mathrm{s}}>0\right)$ and the contact angle $\left(0^{\circ}\right.$ $<\theta<180^{\circ}$ ), the formation free energy of a heterogeneous cluster is always smaller than the free energy of formation of the Kelvin-size cluster in homogeneous nucleation. This is consistent with the general intuition that nucleating on a pre-existing surface reduces the energy spent on surface formation. Therefore, if very small seed nanoparticles (smaller than the critical size $n^{*}$ ) are allowed to grow within the host vapor, no condensation will take place until the nanoparticles reach a size comparable to $\mathrm{n}^{*}$ as defined by the degree of supersaturation of the host vapor. Once the metal nanoparticles reach the critical size $n^{*}$ required for vapor condensation, they act as heterogeneous nuclei for the condensation of the vapor and thus, are immediately removed from the vapor phase by the resulting liquid droplets. By varying the value of $S(T)$ of the host vapor, it should be possible to activate different size nanoparticles to act as heterogeneous nuclei for the condensation of the supersaturated vapor. The size of the nucleating particles resulting from the heterogeneous nucleation process can be determined using transmission electron microscopy (TEM), and the size of the original seed nanoparticles can be estimated from the Fletcher equation. These measurements and estimates can provide direct observation of the heterogeneous nucleation of supersaturated vapors on size-selected seed nanoparticles and comparisons with the estimates from the Fletcher and Kelvin equations. These are the specific objectives of the present experiments and the corresponding data analysis.

\section{RESULTS AND DISCUSSION}

The nucleation time profiles of acetonitrile vapor measured at different supersaturation ratios between 1.74 and 2.53 at $265-266 \mathrm{~K}$ are displayed in Figure 2(a). These 


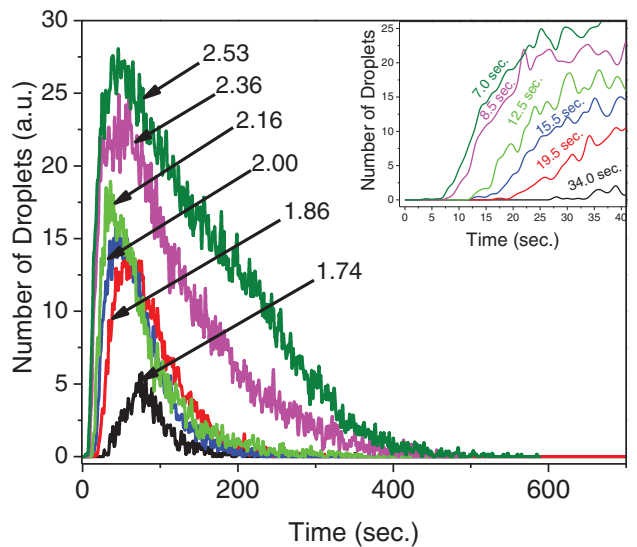

(a)
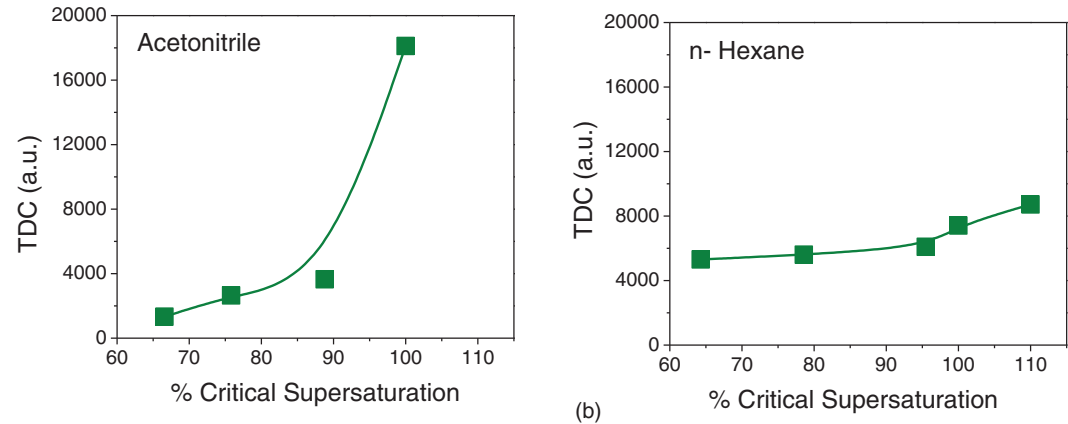

FIG. 2. (a). Time profiles showing the number of acetonitrile droplets condensed on $\mathrm{Al}$ nanoparticles after a single laser pulse $\left(0.56 \mathrm{~W} / \mathrm{cm}^{2}\right)$ at different supersaturations. These experiments were performed at a temperature of maximum supersaturation in the chamber between 265 and $266 \mathrm{~K}$ and a total pressure of 795.0 Torr (He) with no electric field applied between the chamber plates. (b). Total droplet count (TDC) at different supersaturations expressed as percentages with respect to critical supersaturation $\left(S_{c}\right)$ required for the homogeneous nucleation of the liquid $\left(\mathrm{S}_{\mathrm{c}}\right.$ ratios for acetonitrile and $\mathrm{n}$-hexane at $266 \mathrm{~K}$ are 4.01 and 4.20 , respectively). ${ }^{23,26}$

supersaturations correspond to $43 \%$ and $63 \%$, respectively, of the $S_{c}$ required for the homogeneous nucleation of acetonitrile at $\mathrm{T}=266 \mathrm{~K}^{23}$ Therefore, no nucleation is observed before firing the ArF laser on the $\mathrm{Al}$ target inside the DCC. It is also important to exclude the possibility of photoinduced nucleation resulting from the interaction of the $193 \mathrm{~nm}$ photons with supersaturated acetonitrile vapor. This is confirmed by the absence of nucleation events when the laser pulse is fired inside the chamber without hitting the $\mathrm{Al}$ target. Thus the possibility of photoinduced nucleation can be eliminated under the conditions of the heterogeneous nucleation experiments by $\mathrm{Al}$ nanoparticles.

Figure 2(a) shows that as the acetonitrile vapor supersaturation increases, more nanoparticles are activated since the total droplet count (area under each curve) increases. We also observe that the nucleation duration time increases with increasing supersaturation and lasts about $400 \mathrm{~s}$ from the laser vaporization pulse at the highest supersaturation of 2.53 . The inset in Fig. 2(a) shows the onset time of heterogeneous nucleation measured at different supersaturation ratios. The induction time (delay time) is defined as the time elapsed between the generation of the seed nanoparticles and the arrival of the nucleating droplets at the He-Ne laser beam for detection by light scattering. The delay time is measured as 7.0, $8.5,12.5,15.5,19.5$, and $34.0 \mathrm{~s}$ for the supersaturation ratios of acetonitrile of $2.53,2.36,2.16,2.00,1.86$, and 1.74, respectively. It should be noted that the delay time includes the time for laser vaporization/ionization $\left(\sim 10^{-8} \mathrm{~s}\right)$, the time for the seed particles to travel to the nucleation zone $\left(\sim 10^{-2}\right.$ $\left.10^{-3} \mathrm{~s}\right)$, the time for nucleation $\left(\sim 10^{-6} \mathrm{~s}\right)$, and the time for the nucleating droplets to grow and fall across the He-Ne laser beam ( $\sim 0.3-0.4 \mathrm{~s})$ for detection by light scattering.

According to Eq. (2) at small supersaturation ratios, the nucleus size required for condensation is large and because of the slow diffusion of large particles, the induction (delay) time is longer. These results are in excellent agreement with our previous work on the condensation of supersaturated trifluoroethanol vapor on $\mathrm{Mg}$ nanoparticles. ${ }^{12}$ They are also consistent with the results of Mavliev et al., ${ }^{27}$ Chen et al.,${ }^{28}$ and Rosenorn et al. , $^{29}$ where the supersaturations required for activating the nucleating particles were found to decrease with increasing the particles' diameters. Similarly, the work of Seto et al. ${ }^{30}$ on the condensation of dibutyl phthalate vapor induced by seed ions generated by electrospray ionization, showed a significant reduction in supersaturation with increasing the size of the seed ions.

The total droplet counts (TDC) measured for the condensation of acetonitrile and n-hexane vapors at supersaturations expressed as percentages with respect to the critical supersaturation for homogeneous nucleation of each vapor are shown in Figure 2(b). The sharp increase in the TDC of acetonitrile at higher supersaturations reflects the contribution of ion-induced nucleation by charged $\mathrm{Al}$ nanoparticles. The high polarity of acetonitrile molecules (dipole moment $=3.9 \mathrm{D}$ ) enhances the ion-dipole interaction and therefore, ion-induced nucleation becomes significant. ${ }^{18,19}$ This effect 

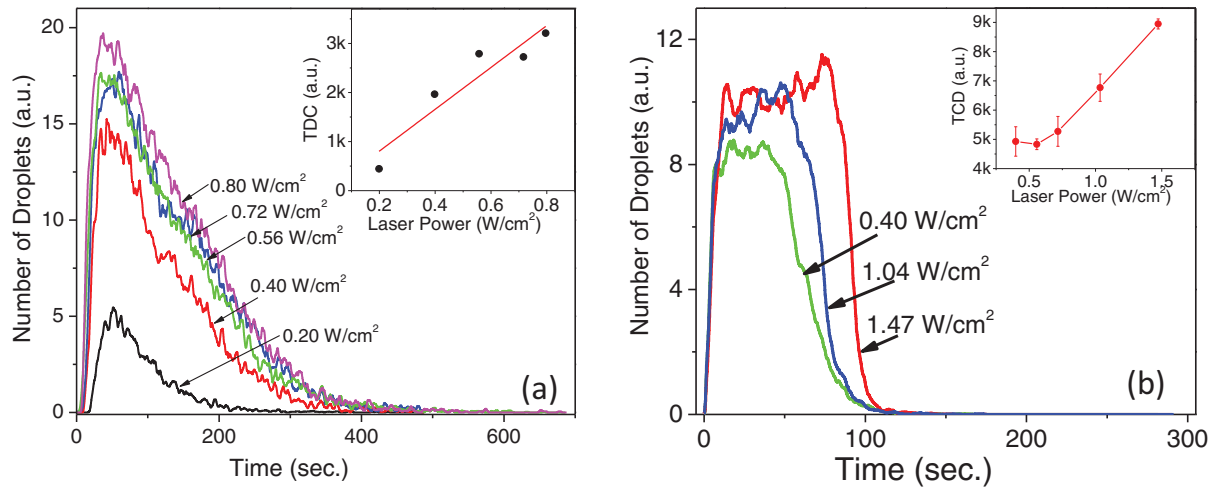

FIG. 3. Time profiles showing the number of acetonitrile (a) and n-hexane (b) droplets condensed on Al nanoparticles after a single laser pulse (ArF, $193 \mathrm{~nm}$ ) at different laser power density $\left(\mathrm{W} / \mathrm{cm}^{2}\right)$ as indicated. Insets show the total droplet count (TDC) as a function of the laser power used for the vaporization/ionization of the $\mathrm{Al}$ target inside the chamber.

is not observed in the case of n-hexane vapor indicating a minor contribution of ion nucleation as a result of the weak ion-induced dipole interaction between the charged $\mathrm{Al}$ nanoparticles and the n-hexane molecules.

Figure 3 illustrates the effect of the laser power on the heterogeneous nucleation of supersaturated acetonitrile and n-hexane vapors on $\mathrm{Al}$ nanoparticles. In both cases, the TDC increases with increasing the laser power. However, the laser power effect is more pronounced in the case of acetonitrile than for n-hexane. This is due to the increased number of charged nanoparticles generated at higher laser powers which results in enhancing the contribution of ion nucleation to the total droplet count. It is also clear that at low laser powers, a significant decrease in the TDC is observed in acetonitrile vapor consistent with the decrease in the number of charged particles generated. In general, most of the nucleation events observed at low laser power can be attributed to neutral $\mathrm{Al}$ nanoparticles. In the case of n-hexane, a significant increase in the laser power is required in order to see an increase in the number of the condensing droplets as shown in Figure 3(b). In this case, at high laser power $\left(1.5 \mathrm{~W} / \mathrm{cm}^{2}\right)$ the number of vaporized atoms increases and hence the number density of the nucleated Al nanoparticles increases as shown in Figure 3(b).

To evaluate the contribution of ion-induced nucleation to the heterogeneous nucleation of the acetonitrile vapor, an electric field is applied across the chamber plates and the droplet count is measured as a function of the applied field $(0$, 50 and $200 \mathrm{~V}$ across the $5.5 \mathrm{~cm}$ height of the DCC) under the same supersaturation and temperature conditions as shown in Figure 4(a). The results clearly show that the largest number of droplets is formed when no electric field is applied across the chamber plates. At zero electric field the Al nanoparticles generated (neutral and charged) diffuse towards the nucleation zone where they can get activated as condensation nuclei depending on the vapor supersaturation and the size distribution of the seed particles in the nucleation zone. However, in the presence of a field, some of the charged particles are removed from the nucleation zone depending on their mobility and are eventually discharged at the chamber plates. At small applied fields, both diffusion and ion mobility control the distribution of the neutral and charged particles, respectively, in the nucleation zone and therefore, some contribution from ion nucleation can be observed depending on the field strength. However, at high applied fields, most of the charged particles are removed from the nucleation zone before vapor condensation can take place and therefore, at high fields most of the nucleation events are due to neutral Al nanoparticles.

Figure 4(b) illustrates the effects of charged particles and the charge sign on the heterogeneous nucleation of acetonitrile at different supersaturation ratios. The peak of the TDC observed in the absence of an applied field reflects contributions from both neutral and charged Al nanoparticles to the heterogeneous condensation of the acetonitrile vapor. The decrease in the TDC by applying either a positive or negative field is due to the removal of the charged Al nanoparticles and therefore at high applied fields $(\sim 200 \mathrm{~V} / 5 \mathrm{~cm})$, the nucleation can be attributed to only neutral Al nanoparticles. The nearly symmetric profile of the TDC dependence on the sign of the applied field indicates that there is no significant sign effect in the heterogeneous nucleation of acetonitrile vapor on charged Al nanoparticles. This is consistent with the large size of Al nanoparticles acting as condensation nuclei especially at lower supersaturation ratios. Similar results have been reported by Chen et al. ${ }^{15}$ who have studied the condensation of water vapor on $\mathrm{SiO}_{2}$ and $\mathrm{TiO}_{2}$ nanoparticles using a flow cloud chamber and did not observe different activation behaviors for particles with different charge signs.
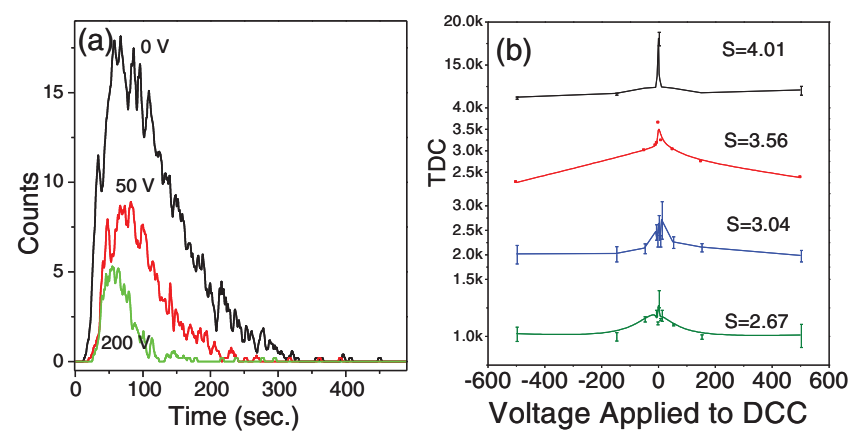

FIG. 4. (a) Time profiles showing the number of acetonitrile droplets condensed on Al nanoparticles after a single laser pulse for different electric fields applied across the DCC plates $(\mathrm{T}=275.1 \mathrm{~K}, \mathrm{~S}=1.74)(\mathrm{b})$ Total droplet count as a function of applied electric field at different vapor supersaturations of acetonitrile $(\mathrm{T}=265-266 \mathrm{~K}$, Total pressure $=815.9,802.6,786.7$, and 774.6 Torr at $\mathrm{S}=4.01,3.56,3.04$, and 2.67, respectively). 


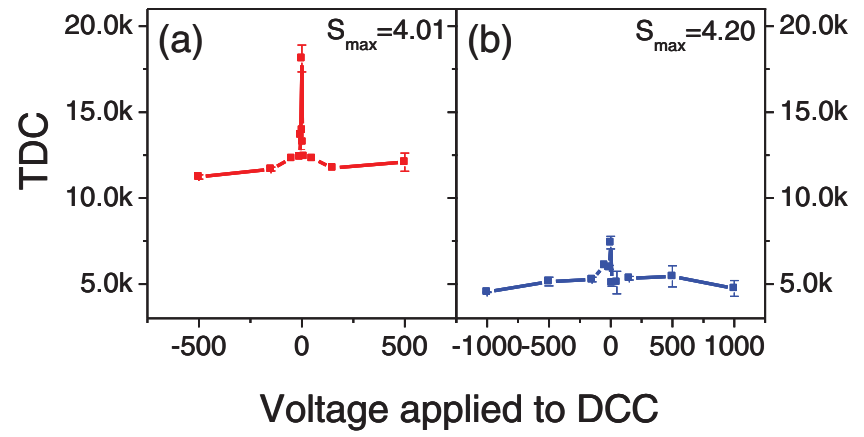

FIG. 5. Total droplet count as a function of applied electric field for acetonitrile (a) and n-hexane (b) at $S(\max )=4.01$ and 4.20, respectively.

To investigate the effect of the dipole moment of the host vapor molecules, the total droplet count is measured as a function of applied electric field for both the acetonitrile and nhexane nucleation experiments as shown in Figure 5. In these experiments the acetonitrile and the n-hexane vapors are set to nucleate homogeneously at $\mathrm{S}(\mathrm{T})=4.01(265 \mathrm{~K})$ and $\mathrm{S}=4.20$ $(266 \mathrm{~K})$, respectively, ${ }^{23,26}$ before firing a single laser pulse on the $\mathrm{Al}$ target inside the chamber. The increased TDC in the absence of applied field reflects the contribution from ion nucleation on charged $\mathrm{Al}$ nanoparticles and this contribution is significantly higher for acetonitrile than for n-hexane as the host vapor. The higher contribution of ion nucleation in acetonitrile vapor is expected due to the strong charge-dipole interaction in comparison with the nonpolar n-hexane molecules. However, in both cases no charge sign effect is observed as indicated by the similar TDCs measured by applying a negative or positive field to the chamber top plate which corresponds to the removal of positively or negatively charged Al nanoparticles, respectively, from the nucleation zone as shown in Figs. 5(a) and 5(b).

To confirm the formation of $\mathrm{Al}$ nanoparticles and their roles as heterogeneous nuclei for the condensation of supersaturated acetonitrile and hexane vapors, we obtained TEM images of the nanoparticles contained in the liquid droplets that fall into the liquid pool during the condensation of the supersaturated vapors. We also obtained XRD of the nanoparticles after evaporation of the liquids. Figure 6 displays TEM images for the $\mathrm{Al}$ nanoparticles formed in supersaturated acetonitrile ( $\mathrm{S}=4.98$, images $\mathrm{a}$ and $\mathrm{b})$ and hexane vapors $(\mathrm{S}$ $=5.14$, images $\mathrm{c}$ and $\mathrm{d}$ ). It is clear that the Al nanoparticles prepared in both the acetonitrile and hexane vapors are well dispersed and exhibit a narrow size distribution with no evidence of agglomeration. The average particle sizes of the Al nanoparticles are determined from the TEM images as $2.3 \pm 0.5$ and $2.1 \pm 0.6 \mathrm{~nm}$ for the acetonitrile and $\mathrm{n}$-hexane supersaturated vapors, respectively. These results confirm that the size of nanoparticles, unlike the total droplet count or the nucleation rate, is independent of the type of the condensing vapor used in the experiment. This is consistent with the nanoparticles acting as condensation nuclei where the size is only determined by the degree of supersaturation of the host vapor.

In different experiments, we have vaporized the Al target under fixed laser power and host vapor supersaturation ratios in an attempt to control the particle size by controlling the va-
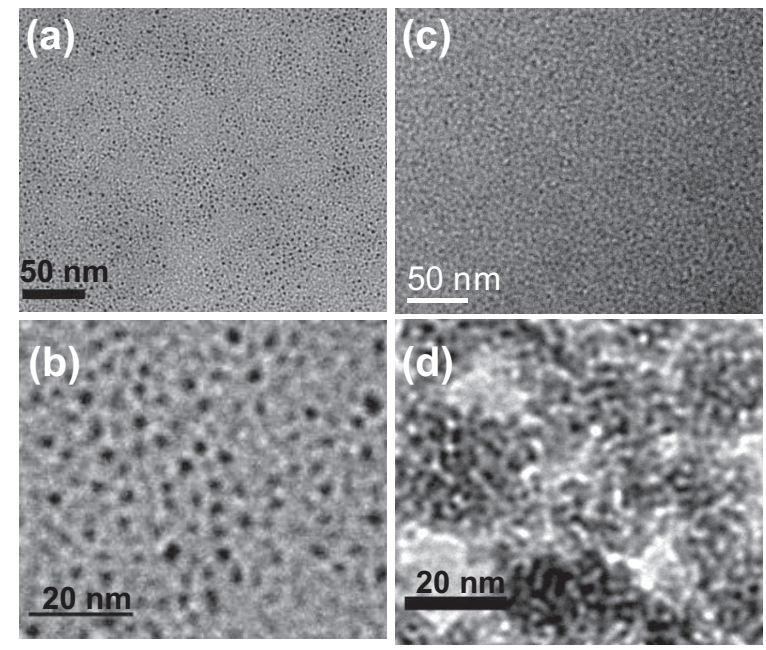

FIG. 6. TEM for Al nanoparticles formed in supersaturated vapors of acetonitrile $(\mathrm{a}, \mathrm{b}, \mathrm{S}(\max )=4.98)$ and $\mathrm{n}$-hexane $(\mathrm{c}, \mathrm{d}, \mathrm{S}(\max )=5.14)$ at $\mathrm{T}=263 \mathrm{~K}$.

por supersaturation. In these experiments we set the chamber at well-defined conditions of temperature and pressure while firing the ArF laser on the Al target at a fixed laser power. We used a low repetition rate for the pulsed ArF laser (1 HZ) to give the chamber some time to reach steady state conditions. The liquid pool sample was collected after $2 \mathrm{~h}$ of operation at a fixed maximum supersaturation ratio, and each experiment was repeated twice. Figures 7(a) and 7(b) and 7(c) and 7(d) display TEM images of the Al nanoparticles collected from the condensation of supersaturated acetonitrile vapors with $S(\max )=2.73$ and 4.98 , respectively, at a constant temperature of $263.4 \mathrm{~K}$. The average particle sizes determined from the TEM images (Figures 7(b) and 7(d) corresponding to $\mathrm{S}$ ( $\max )$ of 2.73 and 4.98 , respectively) are $3.5 \pm 0.7$ and 2.3 $\pm 0.5 \mathrm{~nm}$, respectively. The calculated diameters of the condensation nuclei based on Kelvin's equation at the acetonitrile supersaturation ratios of 2.73 and 4.98 at $263.4 \mathrm{~K}$ are 3.0 and $1.9 \mathrm{~nm}$, respectively. The correlation between the metal nanoparticle's size and the degree of the supersaturation of the condensing vapor is evident. Furthermore, the agreement
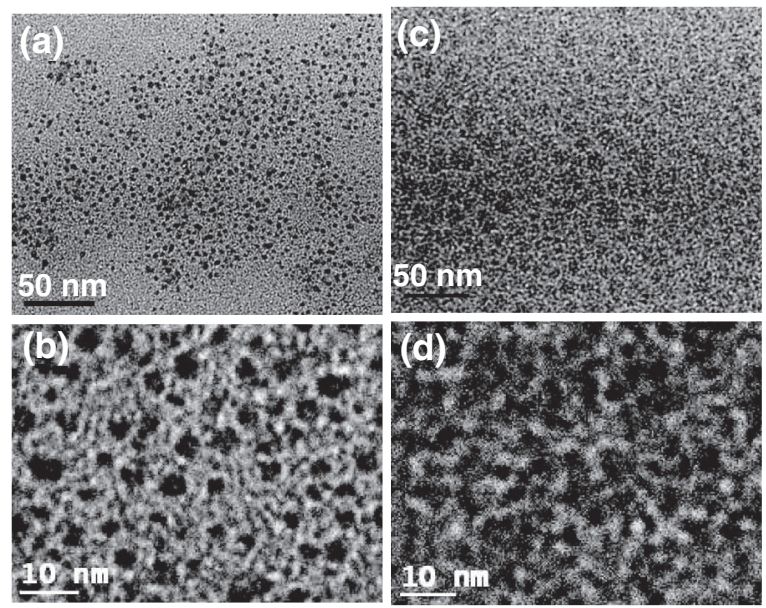

FIG. 7. TEM for $\mathrm{Al}$ at $263 \mathrm{~K}$ nanoparticles formed in two different supersaturated vapors of acetonitrile $(\mathrm{a}, \mathrm{b})$ at $\mathrm{S}(\max )=2.73$ and $(\mathrm{c}, \mathrm{d})$ at $\mathrm{S}(\max )$ $=4.98$. 
TABLE I. Comparison between the Kelvin and Fletcher diameters calculated for supersaturated acetonitrile and hexane vapors and the diameters of the nucleating particles measured by TEM.

\begin{tabular}{lccc}
\hline \hline Vapor & \multicolumn{2}{c}{ Acetonitrile } & n-Hexane \\
\hline Supersaturation $(\mathrm{T}=263.4 \mathrm{~K})$ & 2.73 & 4.98 & 5.14 \\
Kelvin diameter $\left(2 \mathrm{r}^{*}\right)$ & $3.02 \mathrm{~nm}$ & $1.89 \mathrm{~nm}$ & $3.03 \mathrm{~nm}$ \\
Fletcher diameter $\left(2 \mathrm{R}_{\mathrm{F}}\right)$ & $1.52 \mathrm{~nm}$ & $0.85 \mathrm{~nm}$ & $1.37 \mathrm{~nm}$ \\
TEM & $3.5 \pm 0.7$ & $2.3 \pm 0.5$ & $2.1 \pm 0.6$ \\
\hline \hline
\end{tabular}

between the TEM particle size and the calculated Kelvin's diameter is remarkable. A similar correlation has been found between the size of the Al nanoparticles prepared in supersaturated n-hexane vapors and the calculated Kelvin's diameters of the condensation nuclei of n-hexane (see Figure S2 of the supplementary material). ${ }^{25}$ Therefore, it can be concluded that the size of the metal nanoparticles can be controlled under the conditions where they act as critical nuclei for the condensation of supersaturated of host vapors.

The use of pulsed laser vaporization in the present work to generate $\mathrm{Al}$ nanoparticles within a supersaturated organic vapor provides an efficient and controllable approach for the separation of nucleation and growth of the metal nanoparticles. In this approach, the nanosecond laser vaporization pulse generates a highly supersaturated confined plume of aluminum atoms where nucleation occurs very rapidly as the plume propagates within the supersaturated organic vapor. The resulting $\mathrm{Al}$ clusters act as seed heterogeneous nuclei on which the vapor molecules condense to form liquid droplets that fallout from the vapor phase into the liquid pool, and therefore the growth of the $\mathrm{Al}$ clusters is essentially terminated.

To estimate the size of the seed $\mathrm{Al}$ nanoparticles we calculated the Fletcher radius defined by Eq. (9) as the radius of the seed particle which results in the heterogeneous nucleation probability $P=0.5$ as defined by Eq. (8). ${ }^{5,11}$ The equations and parameters used to calculate the Fletcher radius are given in the supplementary material, ${ }^{25}$ and the results are shown in Table I along with the calculated Kelvin diameters and the TEM measured diameters for the nucleating $\mathrm{Al}$ nanoparticles in supersaturated acetonitrile and n-hexane vapors.

The results in Table I show that for the Kelvin diameters of $3.02,1.89$, and $3.03 \mathrm{~nm}$, the Fletcher diameters are $1.52,0.85$, and $1.37 \mathrm{~nm}$, respectively. This indicates that seed nanoparticles with diameters of 1 and $2 \mathrm{~nm}$ are capable of acting as heterogeneous nuclei for the condensation of supersaturated acetonitrile vapor at $\mathrm{S}(\max )$ values of 4.98 and 2.73, respectively, at $263 \mathrm{~K}$. It is clear that at higher supersaturation, very small seed particles are needed for the activation of heterogeneous nucleation.

The comparison between the Kelvin and Fletcher diameters in Table I indicates that for the heterogeneous nucleation of both acetonitrile and hexane vapors, particles are activated at significantly smaller sizes than predicted by the Kelvin equation. This finding is in excellent agreement with the heterogeneous nucleation experiments performed in n-propanol vapor. ${ }^{5,11}$

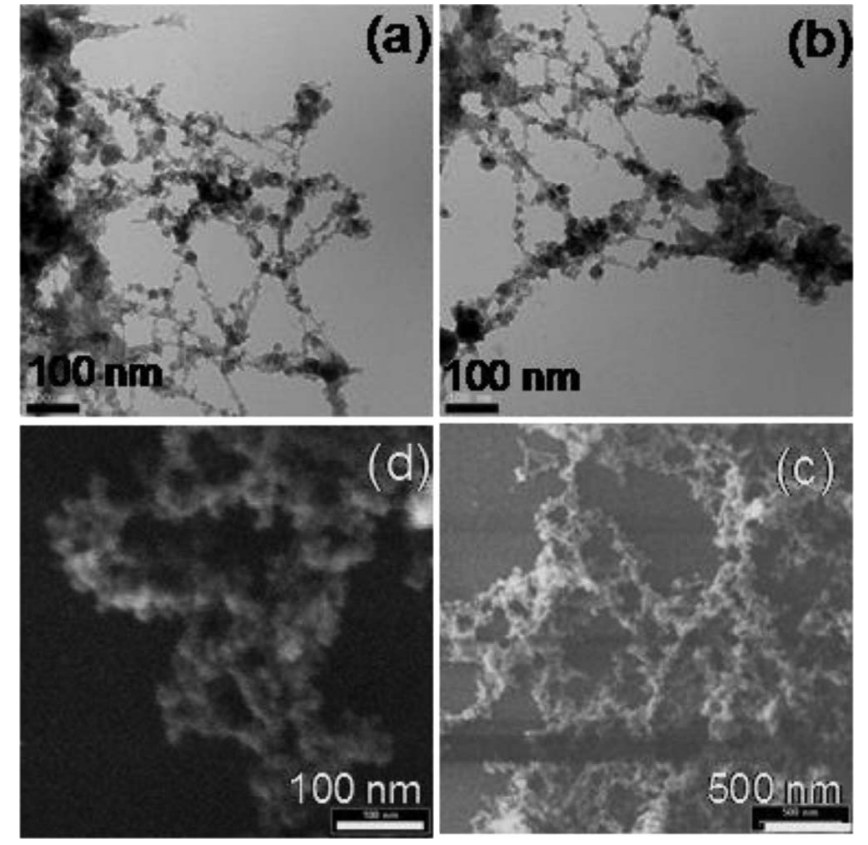

FIG. 8. TEM (a, b) and SEM (c, d) images of Al nanoparticles prepared by laser vaporization (ArF laser, $193 \mathrm{~nm}$ ) of an Al target in pure He carrier gas (800 Torr) at $260 \mathrm{~K}$.

The XRD patterns for the Al nanoparticles formed within the supersaturated acetonitrile and n-hexane vapors and in pure helium without a supersaturated vapor are shown in Figures $\mathrm{S} 3$ and $\mathrm{S} 4$ of the supplementary material..$^{25}$ In all cases, the diffraction patterns match that of the cubic crystal structure of aluminum metal with no evidence for diffraction peaks due to aluminum oxide. This confirms that the Al nanoparticles act as condensation nuclei for the supersaturated organic vapors with no evidence for a chemical reaction or oxide formation taking place under the experimental conditions in the DCC.

To confirm that the formation of size-selected $\mathrm{Al}$ nanoparticles and the lack of nanoparticles' agglomeration are consequences of the nanoparticles acting as condensation nuclei, we prepared $\mathrm{Al}$ nanoparticles using the LVCC method but without the presence of the supersaturated vapor. The TEM and scanning electron microscopy (SEM) images of the resulting $\mathrm{Al}$ nanoparticles are shown in Figure 8. It is clear that in the absence of a supersaturated vapor, the $\mathrm{Al}$ nanoparticles grow into much larger sizes with a broad size distribution and a significant degree of aggregation as shown in Fig. 8. This indicates that the degree of supersaturation of the organic host vapor is the key parameter in activating the $\mathrm{Al}$ nanoparticles to act as heterogeneous nuclei for the condensation of the vapor. The supersaturated vapors condense on the $\mathrm{Al}$ nanoparticles forming liquid droplets that fall into the liquid pool on the bottom plate of the DCC, and the Al nanoparticles become well-dispersed within the liquid with no possibility of growing into larger and aggregated particles.

\section{CONCLUSIONS}

In summary, direct observation and separation of sizeselected $\mathrm{Al}$ nanoparticles acting as heterogeneous nuclei for the condensation of supersaturated vapors of both polar and 
nonpolar molecules have been demonstrated. The results provide new insights on the condensation of supersaturated vapors on nanoparticles including the effects of charged particles, charge sign, and polarity of the condensing molecules on the thermodynamics and kinetics of the heterogeneous nucleation process. Aluminum seed nanoparticles with diameters of 1 and $2 \mathrm{~nm}$ are capable of acting as heterogeneous nuclei for the condensation of supersaturated acetonitrile and hexane vapors. The comparison between the Kelvin and Fletcher diameters indicates that for the heterogeneous nucleation of both acetonitrile and hexane vapors, particles are activated at significantly smaller sizes than predicted by the Kelvin equation. The activation of the $\mathrm{Al}$ nanoparticles occurs at nearly $40 \%$ and $65 \%$ of the onset of homogeneous nucleation of acetonitrile and hexane supersaturated vapors, respectively. The lower activation of charged $\mathrm{Al}$ nanoparticles in acetonitrile vapor is due to the charge-dipole interaction which results in rapid condensation of the highly polar acetonitrile molecules on the charged $\mathrm{Al}$ nanoparticles. The charge-dipole interaction decreases with increasing the size of the $\mathrm{Al}$ nanoparticles and therefore at low supersaturations, most of the heterogeneous nucleation events are occurring on neutral nanoparticles. No sign effect has been observed for the condensation of the organic vapors on the positively and negatively charged Al nanoparticles.

The present approach of generating metal nanoparticles by pulsed laser vaporization within supersaturated organic vapors allows for efficient separation between nucleation and growth of the metal nanoparticles and consequently controls the average particle size, particle density, and particle size distribution within the liquid droplets of the condensing vapor. Strong correlation is found between the metal nanoparticle's size and the degree of the supersaturation of the condensing vapor. This result and the agreement among the calculated Kelvin diameters and the size of the nucleating $\mathrm{Al}$ nanoparticles determined by TEM provide strong proof for the development of a new approach for the separation and characterization of heterogeneous nuclei formed in organic vapors. These processes can take place in the atmosphere by a combination of several organic species including polar compounds which could be very efficient in activating charged nanoparticles and cluster ions of atmospheric relevance.

\section{ACKNOWLEDGMENTS}

The authors acknowledge financial support from the National Science Foundation (Grant No. CHE-0911146).
${ }^{1}$ D. Kashchiev, Nucleation: Basic Theory with Applications (ButterworthHeinemann, Burlington, MA, 2000).

${ }^{2}$ K. F. Kelton and A. L. Greer, Nucleation in Condensed Matter: Applications in Materials and Biology (Pergamon, Amsterdam, The Netherlands, 2010).

${ }^{3}$ D. W. Oxtoby, J. Phys.: Condens. Matter 4, 7627 (1992).

${ }^{4}$ S. Auer and D. Frenkel, Nature (London) 409, 1020 (2001).

${ }^{5}$ P. M. Winkler, G. Steiner, A. Vrtala, H. Vehkamaki, M. Noppel, K. E. J. Lehtinen, G. P. Reischl, P. E. Wagner, and M. Kulmala, Science 319, 1374 (2008).

${ }^{6}$ B.-S. Lee, G. W. Burr, R. M. Shelby, S. Raoux, C. T. Rettner, S. N. Bogle, K. Darmawikarta, S. G. Bishop, and J. R. Abelson, Science 326, 980 (2009).

${ }^{7}$ R. J. Davey, S. L. M. Schroeder, and J. H. ter Horst, Angew. Chem., Int. Ed. 52, 2166 (2013).

${ }^{8}$ N. H. Fletcher, J. Chem. Phys. 29, 572 (1958).

${ }^{9}$ M. C. Weinberg, J. Chem. Phys. 92, 7515 (1990).

${ }^{10}$ M. C. Weinberg, J. Chem. Phys. 96, 9144 (1992).

${ }^{11}$ P. Winkler, A. I. Hienola, G. Steiner, G. Hill, A. Vrtala, G. Reischl, M. Kulmala, and P. E. Wagner, Atmos. Res. 90, 187 (2008).

${ }^{12}$ V. Abdelsayed and M. S. El-Shall, J. Chem. Phys. 126, 024706 (2007).

${ }^{13}$ L. A. Sgro and J. F. De La Mora, Aerosol Sci. Technol. 38, 1 (2004).

${ }^{14}$ P. E. Wagner, D. Kaller, A. Vrtala, A. Lauri, M. Kulmala, and A. Laaksonen, Phys. Rev. E. 67, 021605 (2003).

${ }^{15}$ C.-C. Chen and C.-J. Tao, J. Chem. Phys. 112, 9967 (2000).

${ }^{16}$ F. Chirot, P. Labastie, S. Zamith, and J.-M. L'Hermite, Phys. Rev. Lett. 99, 193401 (2007)

${ }^{17}$ J. L. Katz, J. A. Fisk, and V. M. Chakarov, J. Chem. Phys. 101, 2309 (1994).

${ }^{18}$ D. Kane, G. M. Daly, and M. S. El-Shall, J. Phys. Chem. 99, 7867 (1995).

${ }^{19}$ D. Kane, M. Rusyniak, S. P. Fisenko, and M. S. El-Shall, J. Phys. Chem. 104, 4912 (2000).

${ }^{20}$ S. P. Fisenko, D. B. Kane, and M. S. El-Shall, J. Chem. Phys. 123, 104704 (2005).

${ }^{21}$ M. S. El-Shall, in Nanomaterials: Processing and Characterization with Lasers, edited by S. C. Singh, H. B. Zeng, C. Guo, and W. P. Cai (WileyVCH, Verlag GmbH and Co. KGaA, 2012), Chap. 4, pp. 167-201.

${ }^{22}$ M. S. El-Shall, V. Abdelsayed, Y. B. Pithawalla, E. Alsharach, and S. C. Deevi, J. Phys. Chem. B 107, 2882 (2003).

${ }^{23}$ D. Wright, R. Caldwell, C. Moxely, and M. S. El-Shall, J. Chem. Phys. 98, 3356 (1993).

${ }^{24}$ M. Rusyniak, V. Abdelsayed, J. Campbell, and M. S. El-Shall, J. Phys. Chem. B 105, 11866 (2001).

${ }^{25}$ See supplementary material at http://dx.doi.org/10.1063/1.4890726 for the description of the thermal Diffusion Cloud Chamber (DCC) and details of its operation (Figure S1), TEM images of Al nanoparticles used as heterogeneous nuclei for the condensation of supersaturated n-hexane vapor (Figure S2) and XRD patterns of $\mathrm{Al}$ nanoparticles prepared in supersaturated acetonitrile and n-hexane vapors, and in pure helium carrier gas (Figures S3 and S4). Also, the calculations of the Fletcher diameters for the heterogeneous nucleation of supersaturated acetonitrile and $n$-hexane vapors on aluminum nanoparticles are included.

${ }^{26}$ J. L. Katz, J. Chem. Phys. 52, 4733 (1970).

${ }^{27}$ R. Mavliev, P. K. Hopke, H. C. Wang, and D. W. Lee, J. Phys. Chem. B 108, 4558 (2004).

${ }^{28}$ C. C. Chen, C. J. Tao, and H. C. Cheng, J. Colloid Interface Sci. 255, 158 (2002).

${ }^{29}$ T. Rosenorn, G. Kiss, and M. Bilde, Atmos. Environ. 40, 1794 (2006).

${ }^{30}$ T. Seto, K. Okuyama, L. de Juan, and J. F. de la Mora, J. Chem. Phys. 107, 1576 (1997). 